\title{
Efficacy and Safety of Teneligliptin as Add on Therapy in Indian Type 2 Diabetes Mellitus T2DM Patients Having Dyslipidemia
}

\author{
Parmar Vinendra $\mathbf{M}^{1 *}$ and Goswami Sunita $\mathrm{S}^{2}$ \\ Department of pharmacology, Gujarat Technological University, India
}

Submission: April 10, 2020; Published: August 12, 2020

*Corresponding author: Parmar Vinendra M, Ph. D scholar, Department of pharmacology, L. M. College of pharmacy, Gujarat Technological University, Ahmedabad, Gujarat, India

Abstract

Objectives: The purpose of this study was to investigate the efficacy and safety of teneligliptin, a completely unique and highly selective DPP4 inhibitor in type 2 diabetes mellitus (T2DM) patients having dyslipidemia who are inadequately controlled by relevant conventional therapy in India.

Methods: Study protocol was approved by Institutional Ethics Committee. Diabetic patients having dyslipidemia (male/female) were randomized to receive treatments in two groups, namely conventional therapy [treatment (A)] and add on teneligliptin 20 mg with conventional therapy [treatment (B)] for 24 weeks. Predesigned case report form (CRF) was used to collect information from the prescribing physicians regarding the efficacy and safety of teneligliptin. Efficacy variables included change in serum glycaemic, lipid, and cytokines (IL-6, TNF- $\alpha$ and adiponectin) levels from baseline to week 24. Treatment-emergent adverse events (TEAEs) were also assessed.

Results: A complete of 120 T2DM patient having dyslipidemia were analysed using graph pad prism. Teneligliptin, as add on therapy to conventional therapy significantly reduced serum lipid profile (TC, TG, and LDL) as well as glycaemic parameters (HbA1c, FBG, and PPBG) along with significant rise in serum adiponectin levels as compared to conventional therapy.

Conclusion: Add- on therapy with teneligliptin was found superior over convetional therapy in term of significantly reduced glycemic as well as lipid profile. Further, it was found safe and well tolerated in T2DM patients having dyslipidemia.

Keywords: Teneligliptin; DDP-4 inhibitor; Metformin; Total Cholesterol; Glycated haemoglobin; Adiponectin; Type 2 diabetes mellitus; Dyslipidemia

Abbreviations: NCD: Non-Communicable Disease; IDF: International Diabetes Federation; T2DM: Type 2 Diabetes Mellitus; DPP-4: Dipeptidyl Peptidase-4; GLP-1: Glucagon-like Peptide-1; HbA1c: Glycated Haemoglobin; IEC: Institutional Ethics Committee; DCGI: Drugs Controller General of India; BMI: Body Mass Index; FBG: Fasting Plasma Glucose; PPBG: Post Prandial Blood Glucose; AE: Adverse Event; SAP: Sample Analysis Plan; SD: Standard Deviation

\section{Introduction}

Diabetes mellitus (DM) is found to be related to alterations in lipid metabolism in term of high total cholesterol (TC), triglycerides (TG), low density lipoprotein (LDL) and low high density lipoprotein (HDL), that ends up in dyslipidemia and worsens the prognosis of diabetic patients having atherosclerosis and Cardiovascular disorder(CVD) [1]. The International Diabetes Federation (IDF) estimates the whole number of diabetic subjects is to rise to 69.9 million by the year 2025 [2]. The population of diabetes is rapidly growing because of the expansion of population, urbanisation, ageing, and increasing prevalence of obesity and morbidity [3].
Dyslipidemia, a long-time established risk factor for CVD has effect on $50 \%$ of diabetes patients as compared to nondiabetic population [4]. In DM patient's insulin deficiency or resistance activates intracellular hormone-sensitive lipase which increases the discharge of non-esterified fatty acids (NEFA) from triglycerides that's stored in centrally distributed adipose tissue. High circulating levels of NEFA also increase hepatic triglyceride production [5,6]. Lower levels of adiponectin are considered as an independent risk factor for developing Type 2 DM, dyslipidemia, and cardiovascular diseases [7]. 
A recent statement from one amongst the meta analysis report for the standards of medical care in Diabetes by the American Diabetes Association (ADA) has recommended that initially treatment with metformin as monotherapy after inadequate life style modification, followed by sulfonylurea, thiazolidinedione, Dipeptidyl peptidase-4 (DPP-4) inhibitors, sodium-glucose cotransporter 2 inhibitors (SGLT2-i), glucagon like peptide 1 (GLP 1) receptor agonist, or insulin alone or in combination [8]. However, it's still difficult to search out an antidiabetic agent with long-term glucose control, minimal hypoglycaemia, no weight gain with affordable price. The optimum treatment with antidiabetic drugs to get fair glycaemic control should go hand-in-hand with lipidlowering drugs [9,4]. In diabetic patients having dyslipidemia, in keeping with current guideline, statins are preferred as they are well tolerated, efficacious and don't adversely affect glycaemic control [10].

DPP-4 inhibitors are considered as a cornerstone within the management of T2DM due to their robust efficacy and favourable tolerability profile [11]. Our previous study has demonstrated teneligliptin as add on therapy showing better glycemic control as compared to conventional therapy in Indian patients having T2DM [12]. As there is no future study conducted on add on therapy of teneligliptin in diabetic dyslipidemic patients, our study was designed to evaluate efficacy, safety, and tolerability treatment which can led to improvement in the effectiveness of standard therapy in diabetic patients having dyslipidemia in India. Currently only few reports are available on the role of inflammatory biomarkers in type 2 diabetes and folks with impaired lipid metabolism in Indian population. During this manuscript, we have also tried to incorporate the role of inflammatory regulators (IL 6, TNF $\alpha$ and adiponectin) with respect to add on teneligliptin therapy in type 2 diabetic patients having dyslipidemia in Indian population.

\section{Materials and Methods}

\section{Ethics approval}

The protocol of clinical study, informed consent form and relevant essential documents were approved by Institutional Ethics Committee (IEC); Safety, Health and welfare Ethics committee, registered under Drug Controller General of India (DCGI). The study was conducted according to the Ethical principles of Declaration of Helsinki; Good Clinical Practices guidelines issued by the Central Drugs Standard Control Organization (CDSCO), Indian Councils of Medical Research (ICMR).

\section{Study design and procedure}

A prospective, randomized, open label, study to assess the efficacy and safety of teneligliptin as an add-on therapy to conventional treatment in T2DM patients having dyslipidemia. This study was conducted at Jivraj Mehta Hospital and Bakeri Medical Research Centre, Ahmedabad.

\section{Eligibility criteria}

Study protocol was clearly defined for the patients and informed consent was obtained from all patients before to participation. The study included male and female patients with T2DM having dyslipidemia, aged $>18$ years, $\mathrm{HbA}_{1}$ c levels of $>7.0 \%$, and body mass index (BMI) of $20.0-35.0 \mathrm{~kg} / \mathrm{m} 2$ (both inclusive). Patients were excluded if they had serious disease such as kidney, liver, and cerebral stroke, history of severe heart disease or arrhythmias, taking DPP-4 inhibitor other than teneligliptin, taking statin other than atorvastatin and on insulin therapy, pregnant, and history of alcohol and tobacco use.

\section{Intervention}

Eligible patients were randomized in 1:1 ratio to receive either metformin/glimepiride and atorvastatin (treatment A) or metformin/glimepiride, atorvastatin, and add on teneligliptin (Treatment B). Treatment for both the groups remained stable and it included: teneligliptin $20 \mathrm{mg} /$ day, metformin $500 \mathrm{mg} /$ day, glimepiride $2 \mathrm{mg}$ /day and atorvastatin $20 \mathrm{mg}$ /day for 24 weeks. Patient's demographics data, physical and clinical examination, laboratory assessments were documented in predesigned case report form (CRF).

\section{Biochemical parameters}

Lipid profile including serum Total cholesterol (TC), Triglyceride (TG), Low density lipoprotein (LDL), High density lipoprotein (HDL) and Glycemic parameters including serum glycated haemoglobin (HbA1c), fasting blood glucose (FBG) and post prandial blood glucose (PPBG) levels and Inflammatory cytokine levels IL6, TNF- $\alpha$, and adiponectine levels were measured at baseline and at the end of 24 weeks in both the treatment groups.

\section{Methodology}

Serum cholesterol was estimated by cholesterol oxidase method from ROCHE on COBAS INTEGRA 400 WITH Exteranal quality control (EQAS) from BIO-RAD laboratories (USA) internal quality control from ROCHE diagnostic. Serum LDL and HDL were tested by direct non-immunological method on COBAS INTEGRA 400PLUS. Serum Triglyceride was tested by lipase glycerol method. Values of serum lipids were entered into the computer and computer analysis of the data was obtained.

\section{Cytokines estimation}

From each subject, serum were collected and stored at $-80^{\circ} \mathrm{C}$ until further analysis. Serum IL- 6 and TNF- $\alpha$ levels were measured using the enzyme-linked immunosorbent assay (ELISA) according to the manufacturer's instructions (KRISHGEN Biosytems, Mumbai). The assay sensitivity ranges of KRISHGEN Biosytems kits were $3.12-200 \mathrm{pg} / \mathrm{ml}$ for IL-6 and $6.8-500 \mathrm{pg} / \mathrm{ml}$ for TNF- $\alpha$ in serum samples. The ELISA kits were validated with inter- and intra-assay precision. For adiponectin level was measured using 


\section{Current Research in Diabetes \& Obesity Journal}

the enzyme-linked immunosorbent assay (ELISA) according to the manufacturer's instructions (KINESIS Dx). The assay sensitivity ranges of KINESIS Dx kit was 2-34 $\mu \mathrm{g} / \mathrm{ml}$ adiponectine in serum samples. The ELISA kit was validated with inter- and intraassay precision. The (IL-6, TNF- $\alpha$, and adiponectin) cytokines absorbance measured at $450 \mathrm{~nm}$.

\section{Efficacy and safety end points}

The primary efficacy end point was the change in glycemic and lipid parameter from baseline to 24 weeks. Secondary efficacy endpoints include change in inflammatory (IL-6, TNF- $\alpha$, and adiponectin) cytokine levels from baseline to 24 weeks. During the clinical study period, we monitored possible adverse events (AEs), laboratory values, vital sign and physical examination results. Safety aspects were measured by recording AEs including symptomatic assessment by Naranjo causality scale for adverse events [13]. The incidence of $\mathrm{AE}$ in terms of number per patient was calculated based on the number of events, the number of patients and the total observation period.

\section{Sample size and statistical analysis}

The primary end point, difference in mean $\mathrm{HbA1c}$ from baseline to 24 week was assumed $0.5 \%$ and also the standard deviation (SD) of $0.9 \%$ for each treatment group. Based on a power of $80 \%$ and a type I error rate of alpha= 0.05 (2-tailed), a sample size of at least 60 patients per group was required to detect a clinically significant difference between both the groups [14]. Categorical data was presented as absolute number/ percentage of patients while quantitative data was presented as mean \pm standard deviation (SD). Within group comparison was performed using paired t-test based on the distribution of data. Unpaired t-test was used to analyse the quantitative data for between group comparisons. Missing data was handled using Last observation carried forward (LOCF) method. P value of less than 0.05 was considered as statistical significant difference. Data were calculated using Graph Pad prism version 5.0.

\section{Results}

A flow chart is presented showing the disposition of participants throughout the study (Figure 1). Out of 159 screened patients, 132 eligible patients were randomized during this clinical study. Treatment A included 61 patients and treatment B included 63 patients. As per sample analysis plan (SAP), sixty patients in each group were analysed to detect a clinically significant difference between both the groups. Both the groups had similar demographic and clinical characteristic parameters at baseline (Table 1).

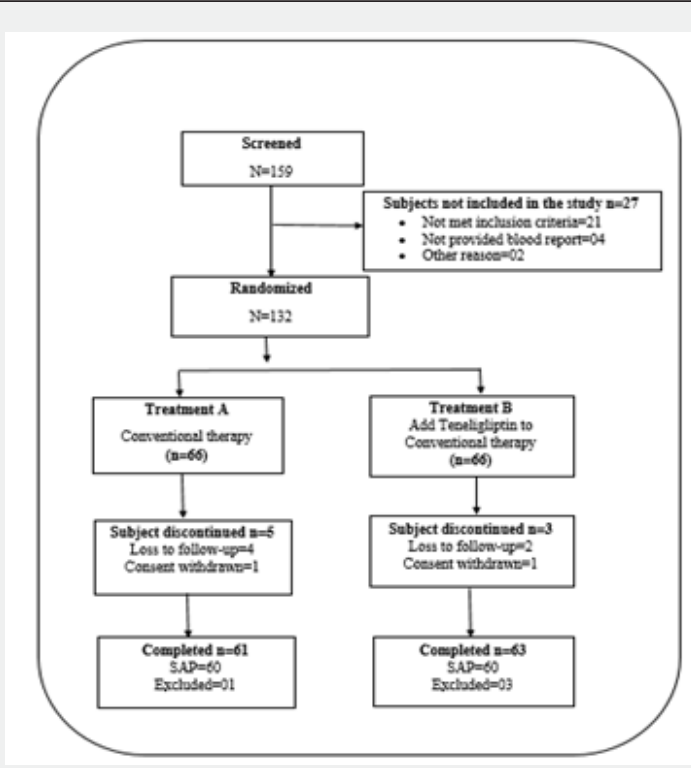

Figure 1: Patient disposition chart.

Table 1: Demographic and clinical characteristic.

\begin{tabular}{|c|c|c|}
\hline Characteristic & Treatment A (N=60) & Treatment B (N=60) \\
\hline Demographic & & $34 / 26$ \\
\hline Gender (Male/Female) & $31 / 29$ & $50.41 \pm 7.27$ \\
\hline Age (year) & $48.56 \pm 8.66$ & $164.21 \pm 8.80$ \\
\hline Height $(\mathrm{cm})$ & $159.9 \pm 10.47$ & \\
\hline
\end{tabular}




\section{Current Research in Diabetes \& Obesity Journal}

\begin{tabular}{|c|c|c|}
\hline Body Weight (kg) & $69.06 \pm 17.79$ & $76.90 \pm 13.40$ \\
\hline Body mass index (BMI) & $25.85 \pm 3.84$ & $28.67 \pm 4.76$ \\
\hline Disease duration (year) & $4.56 \pm 1.61$ & $95.86 \pm 1.50$ \\
\hline Waist (cm) & $92.93 \pm 8.86$ & $102.33 \pm 11.33$ \\
\hline Hip (cm) & $98.63 \pm 10.33$ & $85.96 \pm 13.01$ \\
\hline Pulse /min & $87.16 \pm 13.96$ & $132.97 \pm 18.85$ \\
\hline Systolic blood pressure (mmHg) & $132.9 \pm 18.70$ & $80.28 \pm 10.19$ \\
\hline Diastolic blood pressure (mmHg) & $80.91 \pm 8.66$ & \\
\hline
\end{tabular}

Values are expressed in terms of Mean \pm SD. $N=$ number of patient, $S D=$ standard deviation. Treatment $A$ : Conventional treatment and Treatment B: Add on teneligliptin with conventional treatment

\section{Glycaemic parameters}

$\mathrm{HbA}_{1} \mathrm{c}$ level was found comparable in both the treatment groups at the baseline. However, there was gradual reduction in
$\mathrm{HbA}_{1} \mathrm{c}$ over the period of 24 weeks in both the treatment groups. Between groups comparison showed significant reduction $(\mathrm{p}<0.05)$ in $\mathrm{HbA}_{1} \mathrm{c}$ in treatment $\mathrm{B}$ as compared to treatment $\mathrm{A}$ (Figure 2).

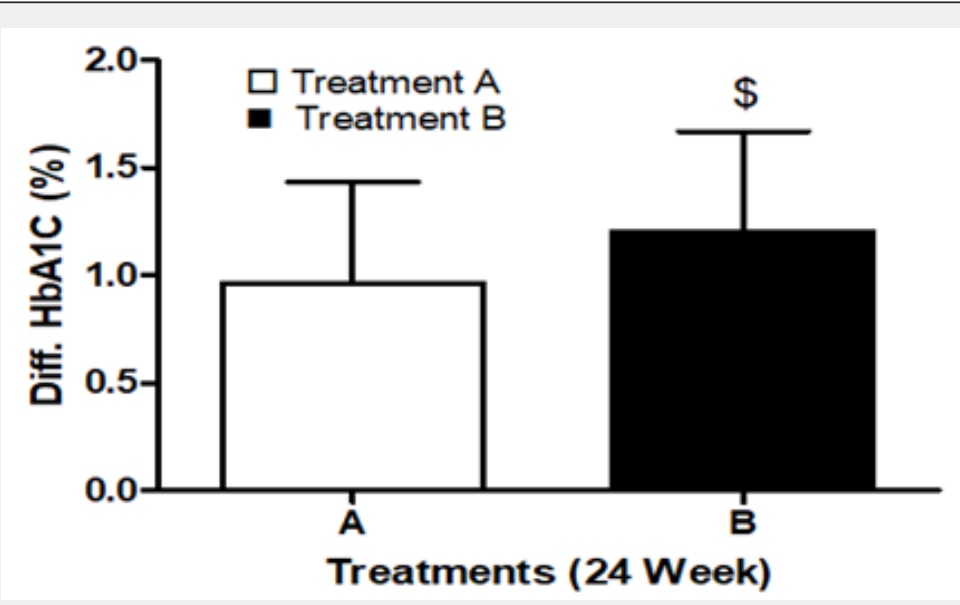

Figure 2: Mean change in Haemoglobin (HbA1c) level.

Data were expressed as the Mean \pm Standard deviation $(n=60)$. \$P value $<0.05$ indicate change indicate change in $\mathrm{HbA} 1 \mathrm{c}$ from baseline to 24 week by unpaired t test (between group comparison).

Blood glucose levels (FBG and PPBG) were found comparable in both the treatment groups at the baseline. However, there were significant reduction in FBG and PPBG levels over a period of 24 weeks in both the treatment groups. However, reduction in glycaemic parameters $\left(\mathrm{HbA}_{1} \mathrm{c}, \mathrm{FBG}\right.$ and $\left.\mathrm{PPBG}\right)$ was statistically significant in treatment B as compared to treatment A after 24 weeks (Table 2). Reduction in mean change of $\mathrm{HbA}_{1} \mathrm{c}$ was $0.96 \pm$ 0.46 and $1.20 \pm 0.45$ in in treatment A \& B respectively. Reduction in mean changes in FBG and PPBG levels were $23.56 \pm 8.40$ and $28.89 \pm 9.17$ in treatment $A$ as compared to $32.62 \pm 11.45$ and $36.62 \pm 9.92$ in treatment B respectively.

Table 2: Mean change in blood lipid and glycemic levels from baseline to 24 weeks after study drug treatments.

\begin{tabular}{|c|c|c|}
\hline Parameters & Treatment A (N=60) & Treatment B (N=60) \\
\hline Total cholesterol (TC) & & $219.00 \pm 14.18$ \\
\hline Baseline (TC) & $218.08 \pm 20.15$ & $166.90 \pm 19.56^{*}$ \\
\hline End of 24 weeks & $176.78 \pm 13.22^{*}$ & $52.10 \pm 20.92 \#(23.78 \%)$ \\
\hline Change in TC & $41.22 \pm 18.57(18.90 \%)$ & \\
\hline
\end{tabular}




\section{Current Research in Diabetes \& Obesity Journal}

\begin{tabular}{|c|c|c|}
\hline Triglyceride (TG) & & \\
\hline Baseline (TG) & $190.88 \pm 18.62$ & $197.36 \pm 18.00$ \\
\hline End of 24 weeks (TG) & $160.13 \pm 18.60^{*}$ & $157.88 \pm 17.30^{*}$ \\
\hline Change in TG & $30.75 \pm 11.56(16.10 \%)$ & $39.48 \pm 15.25 \#(20.00 \%)$ \\
\hline \multicolumn{3}{|c|}{ High density lipoprotein (HDL) } \\
\hline Baseline (HDL) & $36.44 \pm 4.38$ & $37.32 \pm 5.47$ \\
\hline End of 24 weeks (HDL) & $39.08 \pm 4.61^{*}$ & $40.56 \pm 5.53^{*}$ \\
\hline Change in HDL & $2.64 \pm 0.98(7.24 \%)$ & $3.24 \pm 1.08(8.68 \%)$ \\
\hline \multicolumn{3}{|c|}{ Low density lipoprotein (LDL) } \\
\hline Baseline (LDL) & $158.54 \pm 13.15$ & $155.90 \pm 17.39$ \\
\hline End of 24 weeks (LDL) & $113.76 \pm 17.03^{*}$ & $103.09 \pm 17.76^{*}$ \\
\hline Change in LDL & $44.78 \pm 11.61(28.24 \%)$ & $52.80 \pm 15.77 \#(34.06 \%)$ \\
\hline \multicolumn{3}{|c|}{ TC/HDL ratio (atherogenic Index) } \\
\hline Baseline & $6.07 \pm 0.94$ & $6.06 \pm 1.05$ \\
\hline End of 24 weeks & $4.59 \pm 0.69^{*}$ & $4.17 \pm 0.66^{*}$ \\
\hline Change in ration $\mathrm{TC} / \mathrm{HDL}$ & $1.48 \pm 0.53(24.38 \%)$ & $1.82 \pm 0.76(30.03 \%)$ \\
\hline \multicolumn{3}{|c|}{ Glycated heamglobin (HbA1c) } \\
\hline Baseline & $9.63 \pm 1.24$ & $9.45 \pm 1.27$ \\
\hline End of 24 weeks & $8.67 \pm 1.29^{*}$ & $8.24 \pm 1.21^{*}$ \\
\hline Change in HbA1c & $0.96 \pm 0.46(9.96 \%)$ & $1.20 \pm 0.45 \$(12.69 \%)$ \\
\hline \multicolumn{3}{|l|}{ Fasting blood glucose (FBG) } \\
\hline Baseline & $153.54 \pm 20.95$ & $157.53 \pm 19.82$ \\
\hline End of 24 weeks & $129.98 \pm 16.65^{*}$ & $124.91 \pm 18.54^{*}$ \\
\hline Change in FBG & $23.56 \pm 8.40(15.34 \%)$ & $32.62 \pm 11.45 \$(20.70 \%)$ \\
\hline \multicolumn{3}{|c|}{ Post prandial blood glucose (PPBG) } \\
\hline Baseline & $248.46 \pm 27.35$ & $252.82 \pm 27.58$ \\
\hline End of 24 weeks & $219.56 \pm 27.67^{*}$ & $216.19 \pm 25.92^{*}$ \\
\hline Change in PPBG & $28.89 \pm 9.17(11.62 \%)$ & $36.62 \pm 9.92 \$(14.48 \%)$ \\
\hline \multicolumn{3}{|l|}{ Inflammatory cytokines } \\
\hline \multicolumn{3}{|l|}{ IL 6 (pg/ml) } \\
\hline Baseline & $8.027 \pm 0.92$ & $7.927 \pm 0.93$ \\
\hline End of 24 weeks & $7.607 \pm 0.97^{*}$ & $7.409 \pm 0.99 *$ \\
\hline Change in IL 6 & $0.420 \pm 0.38(5.23 \%)$ & $0.518 \pm 0.29(6.43 \%)$ \\
\hline \multicolumn{3}{|l|}{ TNF- $\alpha(\mathrm{pg} / \mathrm{ml})$} \\
\hline Baseline & $15.728 \pm 1.92$ & $15.348 \pm 1.69$ \\
\hline End of 24 weeks & $15.178 \pm 1.85^{*}$ & $14.597 \pm 1.65^{*}$ \\
\hline Change in TNF- $\alpha$ & $0.550 \pm 0.42(3.48 \%)$ & $0.751 \pm 0.42(4.88 \%)$ \\
\hline \multicolumn{3}{|l|}{ Adiponectin $(\mu \mathrm{g} / \mathrm{ml})$} \\
\hline Baseline & $4.441 \pm 0.84$ & $4.673 \pm 0.93$ \\
\hline End of 24 weeks & $5.069 \pm 1.32 *$ & $9.449 \pm 1.44^{*}$ \\
\hline Change in adiponectin & $0.629 \pm 0.87(14.16 \%)$ & 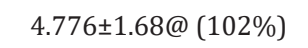 \\
\hline
\end{tabular}

Values are expressed as Mean \pm SD. $N=$ number of patient, $S D=$ standard deviation Treatment A: Conventional treatment and Treatment $B$ : Add on teneligliptin with conventional treatment. * $p<0.05$ from baseline to end of 24 weeks by using paired $t$ test (within group comparison).

$\$ p<0.05$ indicate change in Glycaemic parameter (HbA1c, FBG, and PPBG) from the baseline to 24 weeks.

\# $p<0.05$ indicate change in lipid parameters (TC, TG, and LDL) from the baseline to 24 weeks;

$@ p<0.05$ indicate change in adiponectin level from the baseline to 24 weeks; between groups comparison was done using un-paired $t$ test. 


\section{Current Research in Diabetes \& Obesity Journal}

\section{Lipid parameters}

Lipid parameters were found comparable in both the treatment groups at the baseline. However, there were gradual reduction in Total cholesterol (TC), Triglyceride (TG), atherogenic index (TC/HDL ratio) and Low density lipoprotein (LDL) over the period of 24 weeks in both the treatment groups (paired student t-test). The improvement in lipid profile was significant in treatment B as compared to treatment $A$ at the end of 24 weeks. Mean changes of these parameters have been shown in table 2 .

\section{Total cholesterol levels}

There was significant decrease in serum TC levels after 24 weeks from baseline in both treatment groups. The treatment A reduced the serum concentration of TC from $218.08 \pm 20.15$ $\mathrm{mg} / \mathrm{dL}$ to $176.78 \pm 13.22 \mathrm{mg} / \mathrm{dL}$ and in the treatment B 219.00 $\pm 14.18 \mathrm{mg} / \mathrm{dL}$ to $166.90 \pm 19.56 \mathrm{mg} / \mathrm{dL}$. Further, between groups comparison showed significant reduction in TC in treatment B as compared to treatment A (Figure 3).

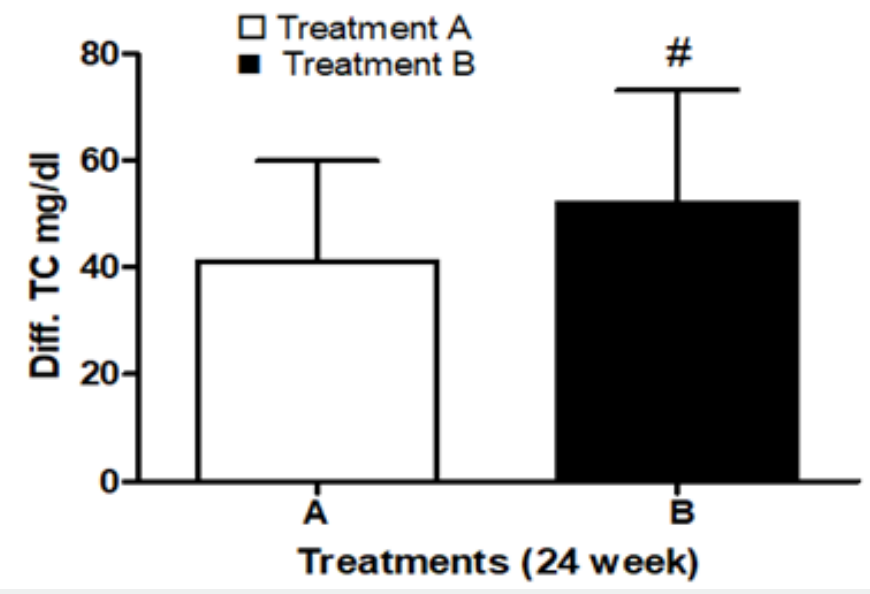

Figure 3: Mean change in total cholesterol (TC) level.

Data were expressed as the Mean \pm Standard deviation $(n=60)$. \# $P$ value $<0.05$ indicate change indicate change in TC from baseline to 24 week by unpaired $t$ test (between group comparison).

\section{Serum triglyceride levels}

Reduction in mean change of TG level was $30.75 \pm 11.56 \mathrm{mg} /$ $\mathrm{dL}$ and $39.48 \pm 15.25 \mathrm{mg} / \mathrm{dL}$ in treatment $\mathrm{A} \& \mathrm{~B}$. The mean change in TG levels significantly increased in treatment B as compared to treatment $A$ at the end of 24 week. Between groups comparison showed significant reduction in TG in treatment $B$ as compared to treatment A (Figure 4).

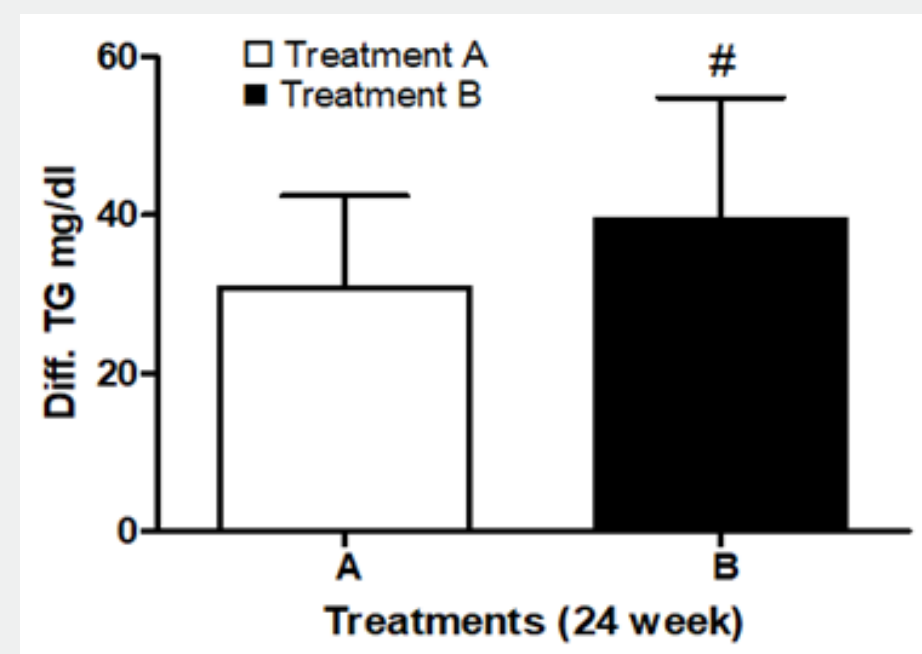

Figure 4: Mean change in Triglyceride (TG) level.

Data were expressed as the Mean \pm Standard deviation $(n=60)$. \# P value $<0.05$ indicate change indicate change in TG from baseline to 24 week by unpaired t test (between group comparison). 


\section{Serum HDL cholesterol}

At the end of 24 weeks, add on teneligliptin group (treatment B) showed tendency to increase HDL level. Improvement in mean change of HDL in treatment A was $7.24 \%$ and $8.68 \%$ in treatment B.

\section{Serum LDL cholesterol levels}

At the end of 24 weeks, add on teneligliptin group (treatment B) showed tendency to reduce in serum LDL levels. Reduction in mean change of LDL in treatment B was $34.06 \%$ as compared to $28.24 \mathrm{mg} / \mathrm{dL}$ in treatment $\mathrm{A}$. The mean change in LDL levels significantly increased in treatment B as compared to treatment
A at the end of 24 week. Between groups comparison showed significant reduction in LDL in treatment $B$ as compared to treatment A.

\section{Cytokines levels}

Serum Adiponectin levels: At the end of 24 weeks, add on teneligliptin group (treatment B) showed tendency to increase adiponectin level. Mean change of adiponectin in treatment B was $4.776 \pm 1.68 \mu \mathrm{g} / \mathrm{ml}$ as compared to $0.629 \pm 0.87 \mu \mathrm{g} / \mathrm{ml}$ in treatment A. The mean change in adiponectin levels significantly improved in treatment B as compared to treatment $A$ at the end of 24 week. Between groups comparison showed better improvement in HDL in treatment B as compared to treatment A (Figure 5).

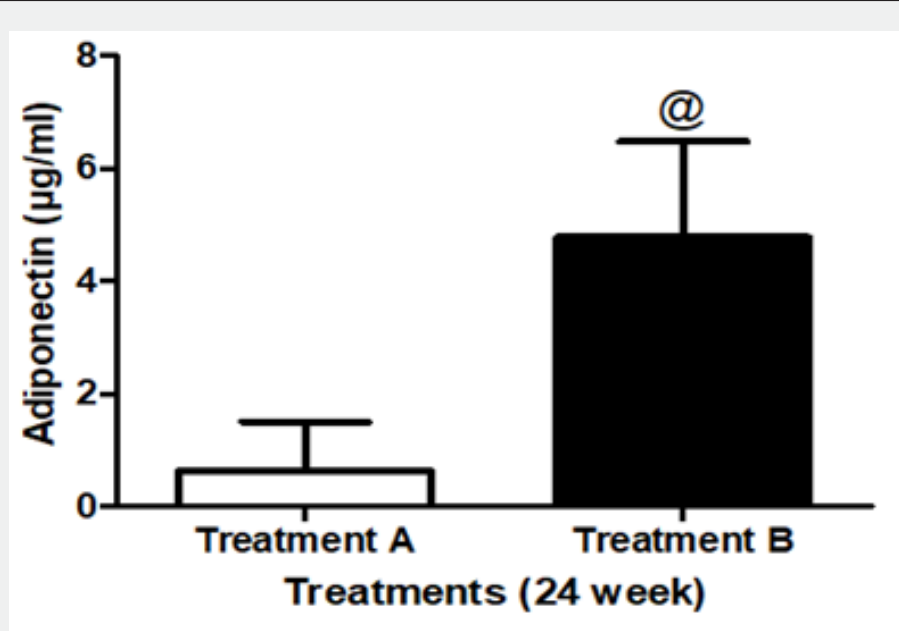

Figure 5: Mean change in adiponectin level.

Data were expressed as the Mean \pm Standard deviation $(n=60)$. @ P value $<0.05$ indicate change indicate change in adiponectin from baseline to 24 week by unpaired $t$ test (between group comparison).

In addition to above, reduction in inflammatory cytokine levels (IL 6 and TNF- $\alpha$ \&) was also observed in treatment B as compared to treatment $A$ at the end of 24 weeks but was not found statistically significant (Table 2).

\section{Safety assessment}

Table 3: Summary of adverse events.

\begin{tabular}{|c|c|c|c|c|}
\hline Adverse Event (AE) & Treatment A N=60 (\%) & Treatment B N=60 (\%) & Naranjo score & Scale \\
\hline Hypoglycemia & $2(3.33 \%)$ & $2(3.33 \%)$ & 9 & Definite \\
\hline Constipation & $5(8.33 \%)$ & $2(3.33 \%)$ & 9 & Definite \\
\hline Abdominal Pain & $6(10.00 \%)$ & $3(5.00 \%)$ & 5 & Probable \\
\hline Acidity & $4(6.66 \%)$ & $3(5.00 \%)$ & 5 & Probable \\
\hline Weakness & $2(3.33 \%)$ & $2(3.33 \%)$ & 4 & Possible \\
\hline Headache & $2(3.33 \%)$ & $1(1.66 \%)$ & 0 & Doubtful \\
\hline Total & $21(35 \%)$ & $13(21.66 \%)$ & & \\
\hline
\end{tabular}

Treatment A: Conventional treatment and Treatment B: Add on teneligliptin with conventional treatment. 


\section{Current Research in Diabetes \& Obesity Journal}

The most common AEs experienced in both the treatment groups were hypoglycaemia, constipation, abdominal pain, acidity, weakness and headache. The incidence of AE was 35\% in treatment A and $21.66 \%$ in treatment B group (Table 3). Revealing better tolerability and safety of teneligliptin under the add on therapy.

\section{Discussion}

Diabetes is progressive disease which include both microvascular and macrovascular complications [15]. The relation between DM and lipid profile has been established during the past decades [16]. Both lipid profile and diabetes are shown to be the important predictors for metabolic disturbances including dyslipidaemia, hypertension, and cardiovascular diseases [4]. Diabetic dyslipidemia accounts for around 80 percent diabetic deaths due to cardiovascular complications [17]. Diabetes is typically managed employing a stepwise approach involving diet and lifestyle modification followed by addition of oral as well as IV antidiabetic drugs [18]. Although international and native guideline recommended lifestyle management as the mainstay of treatment for T2DM, with metformin as the preferred initial oral antihyperglycemic agent in most of the patients, but there is need for extra additional approach synergistic [19].

In the present study, a trial has been made to evaluate the efficacy and safety of newly developed DPP-4 inhibitor, teneligliptin $20 \mathrm{mg}$ in T2DM patients having dyslipidemia who are not adequately controlled by ongoing conventional therapy. The potential effect of teneligliptin in the management of hyperlipidemia and obesity has been established in preclinical studies [20,21]. Dipeptidyl peptidase 4 inhibitors which inhibit the endogenous glucagon like peptide 1 (GLP) metabolism and thereby increases GLP-1 level in the physiological range [22]. They act by regulating insulin and glucagon secretion [23]. Rise in new beta-cells and inhibition of their apoptosis is seen with DPP4 which might be potentially improve the disease pathogenesis [24]. Teneligliptin suppresses proinflammatory activation of macrophages and adipocytes [25]. Therefore, it is a possible target for cardio protective effect.

Present work clearly demonstrated that teneligliptin addition to glimepiride/ metformin stable dose significantly reduced $\mathrm{HbA}_{1} \mathrm{c}$ level as compared to conventional therapy at 24 weeks from the baseline. Further, we also observed significant reduction in blood glucose levels (FBG and PPBG) in both the treatment groups. Results of the present study are found consistent with the previous clinical reports from japan, where efficacy of teneligliptin as add on treatment decreased $\mathrm{HbA}_{1} \mathrm{c}, \mathrm{FBG}$ and PPBG levels at 12 weeks and the same study was expanded to 52 weeks [26]. Our study suggested that, the addition of teneligliptin $20 \mathrm{mg}$ to conventional therapy (metformin/glimepiride) significantly improved the efficacy of conventional therapy. Our study results support the previous clinical study wherein, combination of teneligliptin with that of insulin reduced $\mathrm{HbA}_{1} \mathrm{c}$ level and showed synergistic effect [14]. $\mathrm{HbA}_{1}$ c levels in blood are one of key marker to know glycemic control [27]. In our study, tenligliptin as add on treatment significantly decreased $\mathrm{HbA}_{1} \mathrm{c}$ in patients with T2DM which might be possibly due to its synergistic action.

DM is often related to with alterations in lipid metabolism and abnormalities in serum lipid profile. Dyslipidemia is common in T2DM patients with poorly controlled glycemia [28]. We observed significant decrease in HbA1c and lipid profile at the end of 24 weeks in teneligliptin treated patients. Addition of teneligliptin showed significant reduction in TC at the end of 24 weeks. These findings are supported by one in all the meta-analysis report where in lipid lowering effect of DPP-4 inhibitors revealed a decrease in TC [29].

Beneficial effects of metformin on lipids could be because of inhibition of fatty acids released from adipose tissues, its direct effect on VLDL-C metabolism and/or secondary to enhance insulin sensitivity [30]. Elevated TG levels have been recognized as a risk factor for progression of CVD [31]. In this study, addition of teneliglptin reduced TG levels by $20.02 \%$ at the end of 24 week. Reduction in TG rich lipoprotein may include enhanced expression of the LDL receptors, increased expression of lipoprotein lipase, and reduced expression of apo C-III and very low-density lipoproteins [32]. Thus, teneligliptin might act through either of these mechanism to reduce this.

Besides, add on therapy of teneligliptin showed reduction in LDL-C levels (34.06 \%) in treatment B. Meta-analysis report by Wulffle et al., showed efficacy of metformin in reducing TC, TG and LDL-C in their study with a minimum of 6 weeks of treatment in T2DM patients [33]. So, we conclude that add on therapy of teneligliptin together with metformin might give better reduction in serum LDL levels. Further, mean atherogenic index (TC/HDL ratio) was found comparable from baseline to week 24 for both the treatment groups. The mean atherogenic index decreased $(30.03 \%)$ in treatment B when put next with the treatment A (28.24\%) from baseline data. There is a report of significant decrease in TC, TG and LDL-C with increase in levels of HDL-C after 3 months of treatment with metformin in T2DM patients [34]. Pravin Kumar and Gokul reported a decrease of 16\%, 12\% and $10 \%$ of TC, TG and LDL-C respectively and a $15 \%$ increase of HDL-C; and achieving the lipid control goals with metformin - glimepiride combination therapy of 26 weeks in T2DM in their study [1].

Thus, our results were also parallel with the findings of various reports. Therefore, it will be suggested that teneligliptin addition with conventional antidiabetic therapy can be useful in controlling diabetes with dyslipidemia. Thus our results shown promising data of teneligliptin in T2DM patients with dyslipidemia.

Increased in adipose tissue mass occurs by the expansion of pre-existing adipocytes or by generating new small adipocytes. Chronic overfeeding leads to adipocyte hypertrophy and is related to decrease adiponectin levels and increased IL-6, and TNF- $\alpha$ 
production. In addition, the abnormal functioning of adipocytes like lipodystrophy and inability to store triglycerides and fatty acids may lead to ectopic fat storage in liver, skeletal muscle etc., thus causing dyslipidemia and insulin resistance. This condition can also play an crucial role in the development of a chronic low-grade pro-inflammatory state related to adipose tissue dysfunction and diabetes [35].

Tumor necrosis factor- $\alpha$ and IL-6 are important mediators of inflammation and will provide a possible link between visceral fat and systemic inflammation. They are both known to promote lipolysis and therefore the secretion of free fatty acids, which contribute to a rise in hepatic glucose output and IR, impair adipocyte differentiation, and promote inflammation [36]. Experimental studies and cross-sectional analysis have shown that circulating IL-6 is related to hyperglycemia and insulin resistance. It's also been shown that circulating IL-6 increases with the degree of insulin resistance [37]. The protective effects of adiponectin in the prevention of progression of insulin resistance and in cardiovascular events, and its potent influence in components of the metabolic syndrome, have made it a highly promising therapeutic target [38]. These markers though well understood in terms of their regulation in diabetes population are still lacking acceptance as clinical markers because of the variation of levels among various ethnic groups [39]. Currently limited information is available on inflammatory cytokines in type 2 diabetes and people with impaired glucose and lipid metabolism in Indian population. Our study has demonstrated significantly raised adiponectine levels in treatment B as compared to treatment A. Also, an improvement in adiponectin level found associated with reduction $(p>0.005)$ of inflammatory cytokines (IL-6 \& TNF- $\alpha$ ) which was clearly demonstrated in our study.

\section{Safety assessment}

Clinical symptomatic assessment was done for AEs like hypoglycemia and constipation, which were considered as definite; abdominal pain, and acidity were considered as probable; weakness and headache considered as possible and doubtful respectively by Naranjo AE assessment scale. It is also to be noted that, the incidence of hypoglycemic symptoms was similar 3.33\% in both the groups. For selection of antihyperglycemic agents for add on therapy it include, individual patient characteristic, glucose-lowering efficacy, risk of hypoglycemia, body weight gain, and cardiovascular benefits associated with the drugs are preferentially considered [40].

In summary, results of our study showed that, add on therapy of teneligliptin with standard therapy of anti-diabetic class of drug significantly improved lipid levels by increasing level of good cholesterol as compared to the conventional therapy, this was possibly due to synergistic action of teneligliptin. Hence, during this scenario, the present study supports the initiation of treatment of T2DM with dyslipidemia using teneligliptin, which is affordable and effective in decreasing the glycaemic as well as lipid levels together with lifestyle modifications. Further add on therapy should be initiated if treatment is not satisfactory because optimum glycaemic level is must for the reduction of elevated lipid levels and thereby preventing atherosclerosis and its complications.

\section{Conclusion}

In our study teneligliptin, as add on therapy was found well tolerated and effective in $\mathrm{T}_{2} \mathrm{DM}$ with dyslipidemic patient population. Tenligliptin add on treatment with atorvastatin was found better alongwith conventional-therapy in reducing glycemic and lipid parameters as well as improvement in adiponectin levels in diabetic patients with dyslipidemia.

\section{Acknowledgment}

The authors acknowledge the guidance of Dr.Parag Shah, Dr. Krishna Shah, Dr. Alka Makad, Dr. Chirag Vaghela, Dr. Premal Thakor and Dr. Shubha Desai without their support this work would not have been possible. We also thankful to Krishna shah for providing scientific evaluation and assessment of cytokines. We are also thankful to staff of research department Jivraj Mehta Hospital and Bakeri medical research centre, Ahmedabad for extending required help and research facility for the present study.

\section{Author's Contribution}

The corrospending author has designed the work, data collection, data analysis, statistical analysis, and prepared manuscript. Both authors discussed and provide critical feedback on manuscript.

\section{References}

1. Shashikala E, Motgi S, Raghawa Rao B. N. V., Mohd Abdul Sattar (2020) Study of lipid lowering effects of oral antidiabetic drugs in type 2 diabetes mellitus patients. International Journal of Basic \& Clinical Pharmacology 7(1): 126-132.

2. Sujoy Ghosh, Shailesh Trivedi, Debmalya Sanyal, K D Modi, Sandeep Kharb (2016) Teneligliptin real-world efficacy assessment of type 2 diabetes mellitus patients in India (TREAT - INDIA study). Diabetes Metab Syndr Obes 9: 347-353.

3. Kumar VN, Konyala SR, Bandaru SS, Puchchakayala G (2019) Comparison of Efficacy of Add - on Therapy of Teneligliptin Versus Pioglitazone among Type 2 Diabetes Mellitus Patients Ineptly Controlled on Dual Therapy of Metformin Plus Sulfonylurea. Journal of Diabeteology 76-82.

4. Vijayaraghavan K (2010) Treatment of dyslipidemia in patients with type 2 diabetes. Lipids Health Dis 9: 144.

5. Schofield JD, Liu Y, Rayaz PR (2016) Diabetes Dyslipidemia. Diabetes Ther 7(2): 203-219.

6. Hirano $\mathrm{T}$ (2018) Pathophysiology of Diabetic Dyslipidemia. J Atheroscler Thromb 25(9): 771-782.

7. Ntzouvani A, Fragopoulou E, Panagiotakos D, Pitsavos C (2016) Reduced circulating adiponectin levels are associated with the metabolic syndrome independently of obesity, lipid indices and serum insulin levels : a cross-sectional study. Lipids Health Dis 15(1): 140. 


\section{Current Research in Diabetes \& Obesity Journal}

8. Care D, Suppl SS (2020) American Diabetes Association. Classification and diagnosis of diabetes: Standards of Medical Care in Diabetesd2020. Diabetes Care 43(Suppl 1): S14-S31.

9. Scicali R, Di A, Viviana P, Francesca F, Salvatore U, et al. (2017) New treatment options for lipid - lowering therapy in subjects with type 2 diabetes. Acta Diabetol 55(3): 209-218.

10. Gupta R, Lodha S, Sharma KK, Sharma SK, Gupta S, et al. (2016) Evaluation of statin prescriptions in type 2 diabetes: India Heart Watch-2. 4(1): e000275.

11. Li X, Huang X, Bai C, Qin D, Cao S, Mei Q (2018) Efficacy and Safety of Teneligliptin in Patients With Type 2 Diabetes Mellitus: A Systematic Review and Meta-Analysis of Randomized Controlled Trials. Front pharmacol 9: 449.

12. Parmar V, Goswami S (2019) Efficacy and safety of teneligliptin as add-on therapy to conventional therapy in indian patients with type 2 diabetes mellitus. Asian J Pharm Clin Res 12(12): 116-120.

13. Naranjo CA, Busto U, Sellers EM, Sandor P, Ruiz I, et al. (1981) A method for estimating the probability of adverse drug reactions. Clin Pharmacol Ther 30: 239-245.

14. Kadowaki T, Kondo K, Sasaki N, Miyayama K, Yokota S, et al. (2007) Efficacy and safety of teneligliptin add-on to insulin monotherapy in Japanese patients with type 2 diabetes mellitus: a 16-week, randomized, double-blind, placebo-controlled trial with an open-label period. Expert Opin Pharmacother 18(13): 1291-1300.

15. Young BA, Lin E, Von Korff M, Simon G, Ciechanowski P, et al. (2008) Diabetes complications severity index and risk of mortality, hospitalization, and healthcare utilization. Am J Manag Care 14(1): 1523.

16. Quebec T (2001) Study C. Total Cholesterol/HDL Cholesterol Ratio vs LDL Cholesterol/HDL Cholesterol Ratio as Indices of Ischemic Heart Disease Risk in Men. Arch Intern Med 161: 2685-2692.

17. Adsule SM, Baig MS, Gade PR, Khandelwal PN (2009) A comparative evaluation of safety and efficacy of rosuvastatin, simvastatin and atorvastatin in patients of type 2 diabetes mellitus with dyslipidemia. Int J Diab Dev Ctries 29(2): 74-79.

18. Riddle MC, Bakris G, Blonde L, Boulton AJM, Dalessio D (2018) Standard medical care in diabetes 2018. Diabetes Care 41(1).

19. Snehalatha C, Priscilla S, Nanditha A, Arun R, Satheesh K, et al. (2018) Metformin in prevention of type 2 diabetes. J Assoc Physicians India 66(3): 55-58.

20. Fukuda-Tsuru S, Anabuki J, Abe Y, Yoshida K, Ishii S (2012) A novel, potent, and long-lasting dipeptidyl peptidase-4 inhibitor, teneligliptin, improves postprandial hyperglycemia and dyslipidemia after single and repeated administrations. Eur J Pharmacol 696(1-3): 194-202.

21. Liu H, Li N, Liu Y, Xing J, Feng S, et al. (2017) The dipeptidyl peptidase-4 inhibitor teneligliptin reduces kidney damage from hypercholesterolemia in apolipoprotein E-deficient mice. RSC Adv 7(14): 8702-8708.

22. V Mohan, M Ramu (2019) A Prospectve, Ope N-Label, Randomized Study Comparing EffI cacy and Safety of Teneligliptin VErsus Sitagliptin in Indian Patients with Inadequately Controlled Type 2 Diabetes Mellitus: INSITES Study. Journal of The Association of Physicians of India 67: 14-19.

23. Maladkar M, Sankar S, Kamat K (2016) Teneligliptin: Heralding Change in Type 2 Diabetes. Journal of Diabetes Mellitus 113-131.

24. Hans N (2019) The Efficacy and Safety of Teneligliptin and Metformin versus Glimepiride and Metformin in Patients of Type-2 Diabetes Mellitus Uncontrolled with Monotherapy. Journal of Diabetes and

\section{Metabolism 10(829): 1-9.}

25. Salim HM, Fukuda D, Higashikuni Y, Tanaka K, Hirata Y, et al. (2017) Teneligliptin, a dipeptidyl peptidase-4 inhibitor, attenuated proinflammatory phenotype of perivascular adipose tissue and inhibited atherogenesis in normoglycemic apolipoprotein-E-deficient mice. Vascul Pharmacol 96-98: 19-25.

26. Kadowaki T, Marubayashi F, Yokota S, Katoh M, Iijima H (2015) Safety and efficacy of teneligliptin in Japanese patients with type 2 diabetes mellitus: A pooled analysis of two Phase III clinical studies. Expert Opin Pharmacother 16(7): 971-981.

27.Zeng H, Tong R, Tong W, Yang Q, Qiu M, et al. (2017) Metabolic Biomarkers for Prognostic Prediction of Pre-diabetes: results from a longitudinal cohort study. Sci Rep 7(1): 1-12.

28. Chaudhury D, Aggarwal A (2018) Diabetic Dyslipidemia: Current Concepts in Pathophysiology and Management. Journal of Clinical and Diagnostic Research 12(1): 10-13.

29. Monami M, Lamanna C, Maria C, Edoardo D (2012) DPP-4 Inhibitors and Lipids : Systematic Review and meta- analysis. Adv Ther 29(1): $14-25$

30. Abbasi F, Kamath V, A A Rizvi, M Carantoni, Y D Chen, et al. (1997) Results of a Placebo-Controlled Study of the metabolic effect of the addition of Metformin to Sulfonylurea-Treated Patients evidence for a central role of adipose tissue. Diabet care 20(12): 1863-1869.

31. Jialal I, Singh G (2019) Management of diabetic dyslipidemia: An update. World J Diabetes 10(5): 280-290.

32. Save V, Patil N, Moulik N, Rajadhyaksha G (2006) Effect of Atorvastatin on Type 2 Diabetic Dyslipidemia. Journal of Cardiovascular Pharmacology and Therapeutics 11(4): 262-270.

33. Kooy A, Zeeuw DDE, Stehouwer CDA, Gansevoort RT (2004) The effect of metformin on blood pressure, plasma cholesterol and triglycerides in type 2 diabetes mellitus: A systematic review. Journal of Internal Medicine 1-14.

34. Garimella S, Seshayamma V, Rao HJ, Kumar S, Kumar U (2016) Effect of Metformin on Lipid profile of type II Diabetes. Int J Intg Med Sci 3(11): 449-453.

35. Banerjee G, Upadhyaya S, Kadamkode V, Mahammed R, Doraiswami C (2018) Mediators of inflammation in progression of healthy to type 2 diabetes in Indian population Adiponectin and IL-6. Adipocyte 3: 3945.

36. Indulekha K, Surendar J, Mohan V (2011) High Sensitivity C-Reactive Protein, Tumor Necrosis Factor- $\alpha$, Interleukin-6, and Vascular Cell Adhesion Molecule-1 Levels in Asian Indians with Metabolic Syndrome and Insulin Resistance (CURES-105). J Diabetes Sci Technol 5(4): 982 988.

37. Mohammadi M, Gozashti MH, Aghadavood M (2017) Clinical Significance of Serum IL- 6 and TNF- $\alpha$ Levels in Patients with Metabolic Syndrome. Rep Biochem Mol Biology 6(1): 74-79.

38. Tschritter O, Fritsche A, Thamer C, Haap M, Shirkavand F, et al. (2003) Plasma adiponectin concentra- tions predict insulin sensitivity of both glucose and lipid metabolism. Diabetes 52(2): 239-243.

39. Kazumi T, Kawaguchi A, Sakai K, Hirano T, Yoshino G (2002) Young men with high-normal blood pressure have lower serum adiponectin, smaller LDL size, and higher elevated heart rate than those with optimal blood pressure. Diabetes Care 25(6): 971-976.

40. Moon MK, Hur KY, Ko SH, Park SO, Lee BW, et al. (2017) Combination therapy of oral hypoglycemic agents in patients with Type 2 diabetes mellitus. Diabetes Metab J 41: 357-366. 
This work is licensed under Creative

Commons Attribution 4.0 Licens

DOI: 10.19080/CRDOJ.2020.13.5569

\begin{tabular}{l} 
Your next submission with Juniper Publishers \\
will reach you the below assets \\
- Quality Editorial service \\
- Swift Peer Review \\
- Reprints availability \\
- E-prints Service \\
- Manuscript Podcast for convenient understanding \\
- Global attainment for your research \\
- Manuscript accessibility in different formats \\
( Pdf, E-pub, Full Text, Audio) \\
- Unceasing customer service \\
Track the below URL for one-step submission \\
https://juniperpublishers.com/online-submission.php \\
\hline
\end{tabular}

